\title{
Is the discrimination of motion-path length unique?
}

\author{
WILLIAM EPSTEIN and STEVEN HANSON \\ University of Wisconsin-Madison, Madison, Wisconsin 53706
}

\begin{abstract}
Discrimination of the extent of a motion path may present a special problem since the discrimination calls on memory for changing position and involves pursuit movements of the eye. To determine how these factors affect judgment, discrimination of extents represented by motion paths, successively appearing endpoints, and simultaneously present endpoints was compared under a variety of eye-movement conditions: fixation, pursuit, and saccadic. Discrimination was assessed by the method of adjustment and also by the method of magnitude estimation. Discrimination of motion path extent was found to be as accurate as discrimination of an interspace demarcated by simultaneously presented points or by successively presented points. This was true for brief single presentations of the extents as well as for repeated exposures to the extents. The findings were applied to the analysis of the perception of velocity and the perception of extent.
\end{abstract}

It has been suggested (e.g., Rock, 1975, p. 224) that perceived velocity is mediated by primary discriminations of the length of the movement path (distance traversed) and the time taken to traverse the path. This view postulates a perceptual algorithm for velocity which is isomorphic to the physical definition of velocity: perceived velocity $\left(\mathrm{V}^{\prime}\right)=$ perceived space $\left(\mathrm{S}^{\prime}\right)$ /perceived time $\left(\mathrm{T}^{\prime}\right)$. The algorithm can provide the basis for explaining various aspects of velocity perception. One example is the tendency of perceived velocity to be relatively constant when the distance between the observer and the moving object varies (Brown, 1931; Epstein, 1976; Rock, Hill, $\&$ Fineman, 1968). This tendency toward velocity constancy can be explained by assuming that the perceived length of the movement path and perceived time are unaffected by viewing distance. The algorithm predicts that $\mathrm{V}^{\prime}$ will be constant whenever $\mathrm{S}^{\prime} / \mathrm{T}^{\prime}$ is constant. An experiment to establish this account of speed constancy has been reported by Rock et al. (1968).

Justification of the present study does not rest on acceptance of the above account of velocity perception. Nevertheless, the motive of the present experiments did emerge from consideration of the hypothesis. The algorithm for perceived velocity assumes that the extent of a motion path is readily discriminable and, moreover, that discrimination of motion-path extent does not differ significantly from discrimination of other extents, e.g., that a constancy mechanism operates for motion paths in the same way as it does for other extents. Implicit acceptance of this assumption encouraged Rock, Hill, and Fineman (1968) to test the algorithm by assessing the correlation between perceived velocity and the perceived size of a static continuous extent, instead of the correlation between perceived velocity and the perceived extent of a motion-path.

However, brief consideration discloses that, as a stimulus for the perception of extent, a motion path differs from a static or continuous extent. The cardinal differences between a motion path and a static extent are that (1) a motion path is traced by a moving object and typically elicits pursuit movement of the eyes, while a static extent typically elicits a series of saccades, and (2) information about the length of a motion path must be integrated over time, i.e., discrimination of motion-path length involves memory, while the discrimination of an extent whose endpoints are present simultaneously does not involve memory. The principal aim of the present experiment was to determine whether these two factors affect perception of extent under conditions typical of studies of perceived velocity. Judgments of extent were secured for six types of extents representing the six combinations of two factors: memory load (yes, no) and type of eye movement (pursuit, saccadic, fixation). The results will be applied to the treatments of perceived velocity and perceived extent.

\section{EXPERIMENT 1}

\section{Method}

Subjects. Sixty student volunteers from the University of Wisconsin-Madison each were paid $\$ 2$ for participation as subjects. Ten subjects were assigned to each of the six stimulus conditions.

Stimuli. The standard and comparison displays were generated on the screens on two Tektronix 604 monitors and controlled by an on-line minicomputer (ADS 1800). For presentation to the subjects, the oscilloscope displays were magnified and projected onto two curved rear-projection screens by a folded optical system consisting of a lens and two mirrors. The following six test conditions were arranged: 
(a) Memory load/Pursuit: A trace moved repeatedly back and forth along a horizontal path of $10,21,41$, or $82 \mathrm{~cm}$ in length $\left(1.25^{\circ}-10^{\circ}\right.$ of visual angle) at the rate of $25 \mathrm{~cm} / \mathrm{sec}\left(3.13^{\circ} / \mathrm{sec}\right)$. The trace appeared to the subject to be moving smoothly and continuously. The subject viewed the display under instructions to track the moving trace with his eyes.

(b) No memory load/Pursuit: A trace moved as in Condition a, but the display included two stationary points demarcating the endpoints of the motion path. The endpoints were visible continuously throughout the trial. The subject was instructed to follow the trace with his eyes.

(c) Memory load/Saccadic: Two stationary traces were successively presented each for $1 \mathrm{sec}$. The traces were separated spatially by $10,21,41$, or $82 \mathrm{~cm}$ and separated temporally by an interval equal to the time required to traverse paths of these lengths in the movement displays, i.e., $400,800,1,600$, and $3,200 \mathrm{msec}$ for the four extents, respectively. The subject was instructed to fixate each trace when it was in view and to saccade in the direction of the alternative location immediately upon disappearance of a trace.

(d) No memory load/Saccadic: As in Condition c, two stationary traces were presented, but unlike Condition $c$, the traces were presented simultaneously. The same four spatial separations were used and the subject was instructed to execute repeated saccadic movements between the two locations. The display remained in view for as long as the subject required to complete a judgment.

(e) Memory load/Fixation: The motion display used in Condition a was presented with instructions to fixate an imaginary point in the center of the display. To assist in maintaining fixation, the subject pointed with his unseen outstretched hand at the center of the display and aligned the direction of gaze with the direction of pointing. An arm support was provided and was so positioned that pointing to the prescribed location was facilitated.

(f) No memory load/Fixation: The simultaneous two-trace display used as Condition $\mathrm{d}$ was presented under instructions to fixate midway between the two points.

In addition to the six test displays, two comparison displays were generated. The line comparison was a perceptually continuous horizontally oriented line which could be made to expand or contract in small discrete steps symmetrically about its midpoint. The motion-path comparison was a moving trace whose horizontal path of traverse could be made to expand or contract in small discrete steps symmetrically about its midpoint. The comparison appeared to be moving continuously. The velocity of the comparison trace was $50 \mathrm{~cm} / \mathrm{sec}\left(9.40^{\circ} / \mathrm{sec}\right)$.

The laboratory was totally dark. The projection screens were occluded by opaque flat-black posterboard in which narrow slits were cut to allow for observation of the traces. The intensity of the trace was below $1 \mathrm{~cd} / \mathrm{m}^{2}$ so that neither the borders of the slit aperture nor the immediate surrounding were illuminated. Under these conditions, only the traces were visible in uniformly dark surroundings. The movement paths were at the eye level of the subject. The test display always was located at a distance of $4.57 \mathrm{~m}$ from the subject, the comparison display, at a distance of $3.05 \mathrm{~m}$ from the subject. The two displays were separated widely so that successive inspection of the displays was necessary.

Procedure. Each subject was tested individually on a series of 24 experimental trials. On each trial the subject directed the experimenter to adjust the length of the comparison line or motion-path to match the test extent. A subject was exposed to only one test condition. The first 12 experimental trials were composed of the four test extents presented three times in random order with the comparison mode either line or motion path for all 12 trials. The second set of 12 trials reexposed the test extents in a different order with the alternative comparison mode. The trials were self-paced, providing as many traverses of the standard motion displays and as much inspection time of the test static displays as the subject needed. When the subject was satisfied that a match had been achieved, he pressed a microswitch which terminated the trial and caused the time to respond and the response to be recorded automatically by Teletype. The instructions to the subject stressed phenomenal matching and the importance of conforming to the eye-movement directions while inspecting the standard. Several familiarization trials preceded the first experimental trials.

\section{Results}

Table 1 presents the mean times to complete a judgment. The means are based on the responses for all four extents in each of the six test conditions. Examining the contrasts a vs. b, $c$ vs. d, and e vs. $f$ reveals large effects of memory load on judgment time. In all three cases, completion of the judgment took longer when memory was involved. It was also the case that more time was required for the motion path as response mode than for the line mode. Judgment times also differed as a function of eye movements, with judgment time fastest for tracking movements $(33.63 \mathrm{sec})$, slowest for fixation $(40.55 \mathrm{sec})$, and intermediate for saccadic movement $(37.26 \mathrm{sec})$.

Table 2 reports the mean matches and SDs for the four extents under each of the six test conditions. To facilitate comparison, the means are also presented in Figure 1. The open circles in Figure 1 present the results for the line comparison; the filled circles, for the motion-path comparison. Examination of these data shows that (1) the standard extents were discriminated under all conditions, (2) the means for the two response modes within a test condition were very similar, and (3) there was very little difference among the means for the six test conditions. Analysis of variance yielded a significant main effect associated with extent, $F(3,162)=869.64, p<.001$. The main effects of viewing condition and comparison mode did not approach significance, $p>.25$. There was also a significant Viewing Condition by Comparison Mode by Extent interaction. Examination of Figure 1 discloses the nature of the interaction effect. Under Conditions $d$ and $f$, when the comparison mode was a moving trace, the slopes of the mean matches were flatter than under the remaining conditions. Examination of the individual subject data suggests that this was due to the anomalous behavior of two subjects in each of these conditions. Since these two conditions cannot be segregated from the other conditions on sensible grounds, we propose to ignore this interaction in discussing the results.

Table 1

Mean Time in Seconds to Complete a Trial

\begin{tabular}{lcccccc}
\hline & \multicolumn{6}{c}{ Standard Display Condition } \\
\cline { 2 - 7 } \multicolumn{1}{c}{\begin{tabular}{c} 
Mode \\
\cline { 2 - 7 }
\end{tabular}} & $\mathrm{a}$ & $\mathrm{b}$ & $\mathrm{c}$ & $\mathrm{d}$ & $\mathrm{e}$ & $\mathrm{f}$ \\
\hline Motion Path & 42.24 & 35.04 & 47.22 & 35.46 & 59.04 & 31.92 \\
Line & 35.22 & 22.02 & 39.30 & 27.06 & 39.84 & 31.38 \\
\hline
\end{tabular}


Table 2

Means (centimeters) and SDs of Matches for the Four Standard Extents Presented Under Six Stimulus Conditions

\begin{tabular}{|c|c|c|c|c|c|c|c|c|c|}
\hline \multirow{3}{*}{$\begin{array}{l}\text { Standard } \\
\text { Condition }\end{array}$} & & \multicolumn{4}{|c|}{ Line Comparison } & \multicolumn{4}{|c|}{ Motion Path Comparison } \\
\hline & & \multicolumn{4}{|c|}{ Standard Extent $(\mathrm{cm})$} & \multicolumn{4}{|c|}{ Standard Extent $(\mathrm{cm})$} \\
\hline & & 10 & 21 & 41 & 82 & 10 & 21 & 41 & 82 \\
\hline a & $\begin{array}{l}\text { Mean } \\
\text { SD }\end{array}$ & $\begin{array}{r}13.40 \\
7.61\end{array}$ & $\begin{array}{r}26.14 \\
7.66\end{array}$ & $\begin{array}{l}48.98 \\
12.28\end{array}$ & $\begin{array}{l}76.63 \\
11.09\end{array}$ & $\begin{array}{r}14.72 \\
3.98\end{array}$ & $\begin{array}{r}25.57 \\
8.14\end{array}$ & $\begin{array}{l}51.17 \\
18.48\end{array}$ & $\begin{array}{l}71.14 \\
14.67\end{array}$ \\
\hline $\mathrm{b}$ & $\begin{array}{l}\text { Mean } \\
\text { SD }\end{array}$ & $\begin{array}{r}12.72 \\
3.14\end{array}$ & $\begin{array}{r}23.65 \\
3.59\end{array}$ & $\begin{array}{r}40.53 \\
8.30\end{array}$ & $\begin{array}{l}75.30 \\
12.82\end{array}$ & $\begin{array}{r}10.87 \\
2.62\end{array}$ & $\begin{array}{r}23.28 \\
4.69\end{array}$ & $\begin{array}{l}41.80 \\
13.27\end{array}$ & $\begin{array}{l}74.10 \\
12.87\end{array}$ \\
\hline c & $\begin{array}{l}\text { Mean } \\
\text { SD }\end{array}$ & $\begin{array}{r}11.88 \\
2.87\end{array}$ & $\begin{array}{r}22.05 \\
5.79\end{array}$ & $\begin{array}{r}36.81 \\
5.00\end{array}$ & $\begin{array}{l}60.70 \\
12.14\end{array}$ & $\begin{array}{r}16.29 \\
4.67\end{array}$ & $\begin{array}{l}30.57 \\
11.41\end{array}$ & $\begin{array}{l}44.16 \\
13.09\end{array}$ & $\begin{array}{l}65.91 \\
10.54\end{array}$ \\
\hline d & $\begin{array}{l}\text { Mean } \\
\text { SD }\end{array}$ & $\begin{array}{l}16.52 \\
17.72\end{array}$ & $\begin{array}{r}21.97 \\
8.21\end{array}$ & $\begin{array}{r}35.05 \\
9.61\end{array}$ & $\begin{array}{l}67.48 \\
12.57\end{array}$ & $\begin{array}{r}20.39 \\
9.76\end{array}$ & $\begin{array}{l}32.51 \\
10.10\end{array}$ & $\begin{array}{r}38.37 \\
9.70\end{array}$ & $\begin{array}{l}56.91 \\
20.83\end{array}$ \\
\hline $\mathbf{e}$ & $\begin{array}{l}\text { Mean } \\
\text { SD }\end{array}$ & $\begin{array}{r}13.83 \\
5.54\end{array}$ & $\begin{array}{r}24.31 \\
7.11\end{array}$ & $\begin{array}{r}43.88 \\
9.88\end{array}$ & $\begin{array}{l}69.40 \\
10.64\end{array}$ & $\begin{array}{r}17.54 \\
9.34\end{array}$ & $\begin{array}{r}24.87 \\
6.41\end{array}$ & $\begin{array}{l}44.89 \\
14.25\end{array}$ & $\begin{array}{r}73.55 \\
9.19\end{array}$ \\
\hline f & $\begin{array}{l}\text { Mean } \\
\text { SD }\end{array}$ & $\begin{array}{l}14.58 \\
13.44\end{array}$ & $\begin{array}{l}22.46 \\
13.26\end{array}$ & $\begin{array}{r}34.82 \\
6.49\end{array}$ & $\begin{array}{l}58.58 \\
15.73\end{array}$ & $\begin{array}{l}23.12 \\
11.94\end{array}$ & $\begin{array}{l}34.02 \\
12.18\end{array}$ & $\begin{array}{r}31.85 \\
5.70\end{array}$ & $\begin{array}{r}48.84 \\
9.43\end{array}$ \\
\hline
\end{tabular}

Note-Condition a: moving point, tracking; Condition b: moving between endpoints, tracking; Condition c: successive endpoints, saccadic; Condition d: simultaneous endpoints, saccadic; Condition e: moving point, fixation; Condition f: simultaneous endpoints, fixation.

Is there reason to believe that the special characteristics of the motion path as a stimulus for perception of extent make this discrimination unique? The data do not support an affirmative answer. For the temporal values and angular displacements or

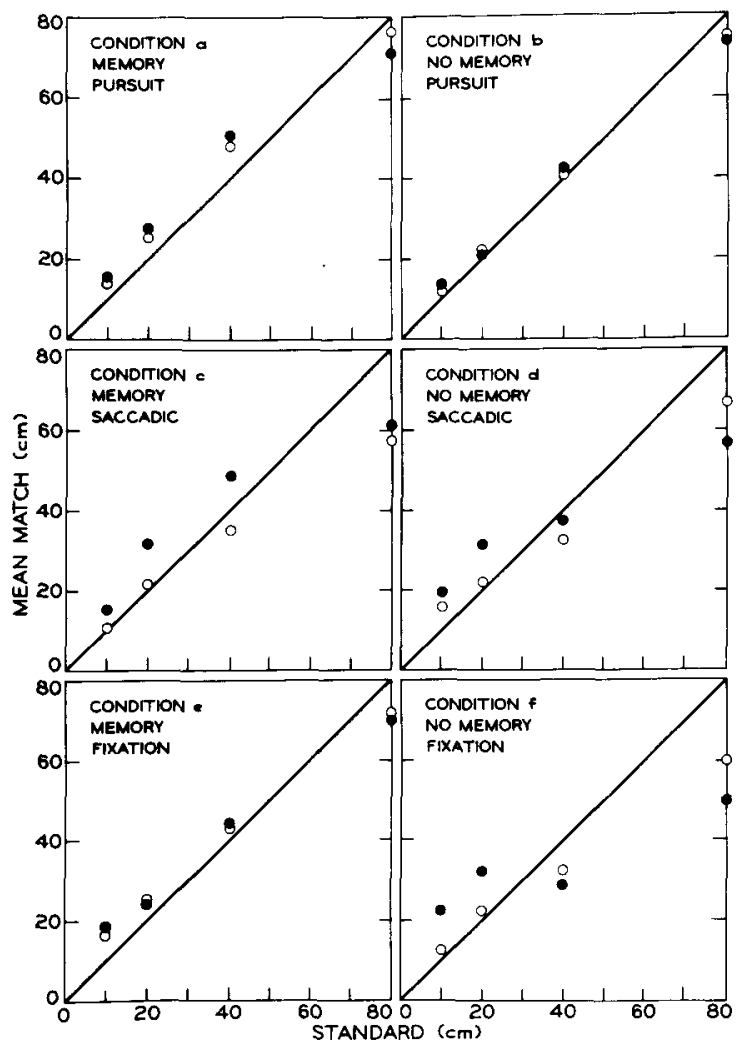

Figure 1. Mean matches under different conditions of standard and comparison displays. (Filled circles are matches for the motion path comparison, open circles are for line comparison.) separations that were sampled, there was no effect of memory or eye movements on perceived extent. Regarding the effect of memory, there are three constrasts, Conditions a vs. b, c vs. d, and e vs. f, which compare performance with and without memory load for the same type of eye movement. None of the three contrasts disclosed an effect of memory. The necessity to store the endpoints of a movement path of the egocentric location of static points in memory did not reduce accuracy of discrimination, nor did the memory requirement increase variability. This is not to say that memory requirements never will affect performance. The retention intervals tested in this study were short and the displays were presented repetitively. Longer retention intervals or brief single presentations might reveal an effect of memory. Support for this latter speculation is provided indirectly by the differences in time to complete a judgment which were associated with the memory load factor. These findings suggest that the subject compensated for imposition of memory load by increasing inspection time. Accordingly, if inspection times for the displays were brief and the same, irrespective of memory requirements, an effect of memory load might be evident.

The influence of eye movements on the perception of extent may be assessed by comparing the judgments for Conditions a vs. b, c vs. d, and e vs. f. These comparisons give no evidence of a role for eye movements. However, inasmuch as the actual eye movements were not monitored, this conclusion can be offered only tentatively. Three practiced observers who served in pilot work reported no difficulty in adopting the three eye-movement modes, but objective data plainly is preferable. 


\section{EXPERIMENT 2}

Experiment 2 differed from Experiment 1 in four respects: (a) An eye-movement monitor was used to provide objective records of eye movements. (b) The test displays was presented briefly; a single cycle in the case of the moving point of the alternating points and an equivalent brief duration for the display of simultaneous endpoints. (c) In Experiment 1, objective velocity was constant for all motion displays regardless of distance traversed; the alternating endpoint displays were presented at ISIs which were equivalent to the respective traverse times. The same arrangement prevailed for one of the two trial blocks in Experiment 2. However, when this arrangement is applied to a single cycle, the extent of the display is confounded perfectly with duration. This potential difficulty was assessed in a second block of trials on which velocity was made proportional to extent traversed so that duration was the same for all extents. (d) Since reliable application of the method of adjustment requires longer than desired exposures of the test display, the adjustment method was replaced by the magnitude-estimation method.

\section{Method}

Subjects. Thirty-six student volunteers from the University of Wisconsin-Madison were each paid $\$ 2$ for participation as subjects. Six subjects were assigned to each of the six test conditions.

Stimuli. The stimuli were abbreviated versions of the displays used in Experiment 1. Six extents, 22.50, 33.35, 44.45, 55.58, 66.68 , and $77.79 \mathrm{~cm}$, were presented under each of the six test conditions. The test display was always located at a distance of $4.57 \mathrm{~m}$ from the subject.

Procedure. Each subject was tested individually with one of the six test types. The session began with an explanation of the procedure of magnitude-estimation and a series of practice applications involving estimations of egocentric (absolute) distance. The procedure followed closely the prescriptions provided by Stevens (1975) in his discussion of the free-modulus variation of the method of magnitude estimation. The distinctive feature of the free method is that no modulus (standard) is presented and no number scale is provided by the experimenter. The subject is instructed to select the number he considers appropriate for the first and all subsequent test stimuli. After the initial practice series was concluded, the eye-movement monitor (Narco-Biometrics Model 200A) was fitted to the subject and the monitor was calibrated. Following five practice estimates of extent, the subject provided 36 extent estimates. The trials were arranged in six randomized blocks, each calling for an estimate of each of the six extents. For half of the subjects, the first three blocks presented constant velocity; for the other subjects, the first three blocks presented constant duration. Eye movements were monitored on one block selected at random from the first three blocks and one block selected randomly from the second set of three biocks. The output of the monitor associated with the subject's horizontal eye movements was presented on a strip chart recorder (Gould Brush 280).

\section{Results and Discussion}

The mean magnitude estimates for the six extents under each test condition are shown in Figure 2.

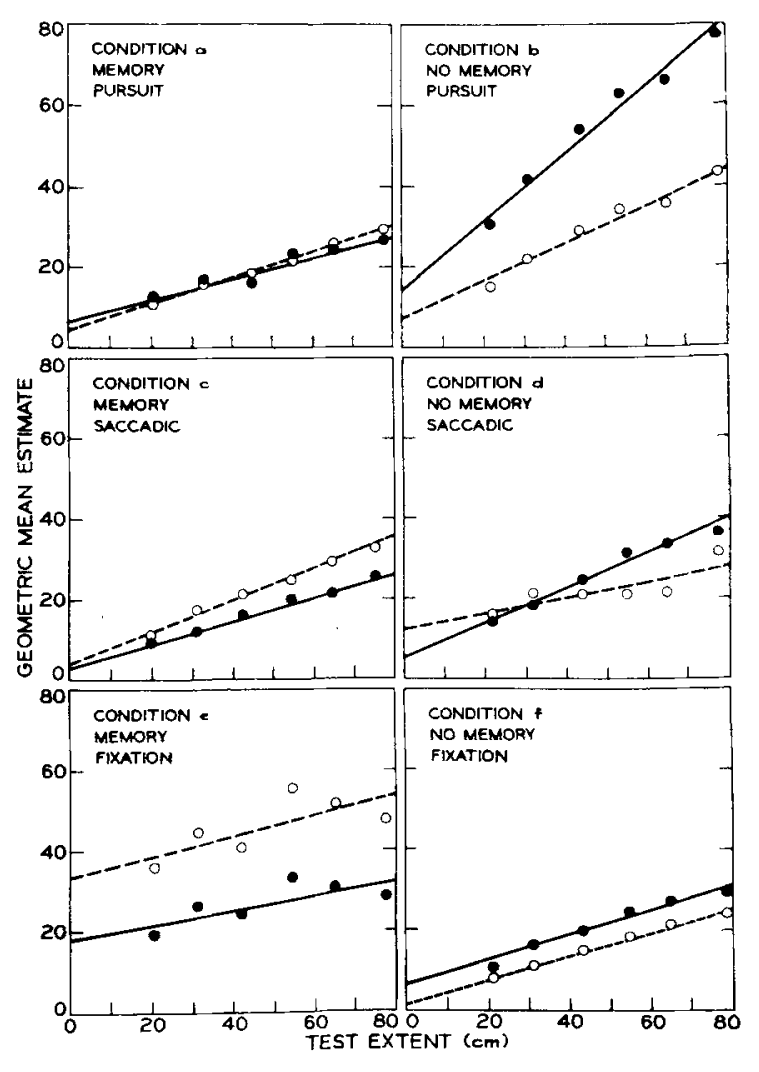

Figure 2. Geometric mean estimates of extent for different conditions of the standard display. (Filled circles are dats for all subjects serving in each condition. Open circles are data for subset of subjects conforming to eye-movement instructions. Solid and broken lines are least squares fit lines for all subjects and the conforming subset, respectively.)

Each panel presents two sets of data: overall, the means computed for all six subjects assigned to the test condition, and conforming, the means for the subset of subjects whose eye-movement records provided unambiguous evidence that the subject had conformed to the eye-movement instructions. There were nonconforming subjects in every group. Typically, the eye-movement records for the nonconforming subjects were erratic and could not be classified confidently into any of our three designated categories. Figure 3 shows the mean magnitude estimates for the constant velocity displays and for the constant duration displays averaged over the six types of test displays. The data from all 36 subjects were included.

Examination of Figures 2 and 3 shows that the main features of the findings of Experiment 1 were reproduced with the very different procedures of Experiment 2. The correlations between responses of conforming and nonconforming subjects in each panel of Figure 2 were very high $(p<.05$, Fisher $z$-transformation significance probability), with the 


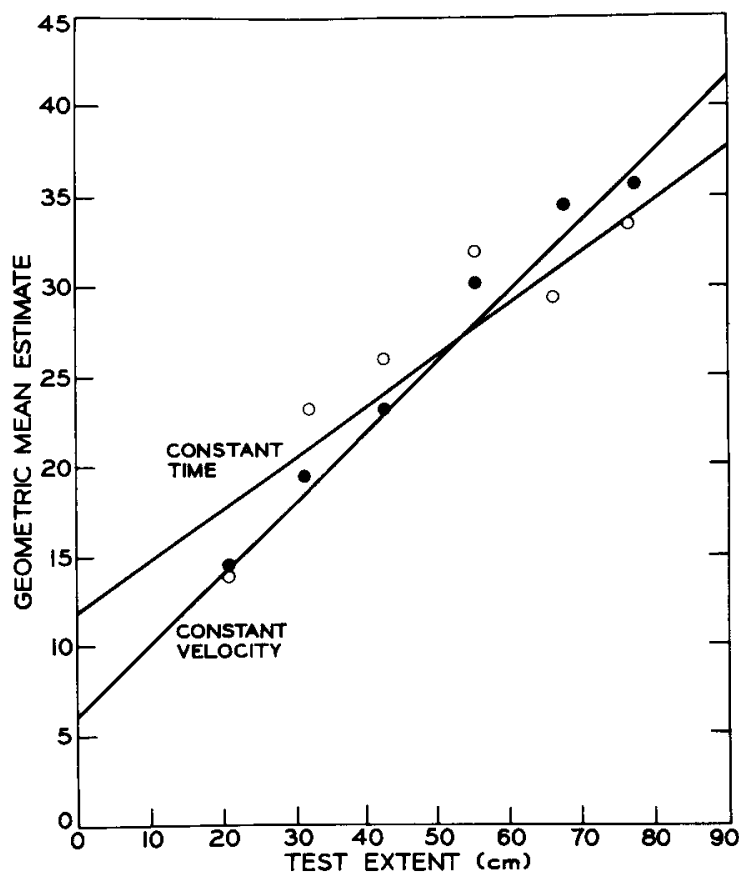

Figure 3. Geometric mean estimates of extent. Filled circles are data for constant velocity condition. Open circles are data for constant time conditions.

single exception of the correlation between conforming and nonconforming subjects for Condition $\mathrm{d}$. In general, the correlations between responses of the subjects in all conditions were also very high. The correlations between conditions were all significant $(p<.01)$, except for the correlation between subjects who conformed to the eye-movement instructions in Condition $\mathrm{d}$ and the conforming and overall responses in Condition $\mathrm{e}$. It is worth noting, however, that only two of the six subjects in Condition $\mathrm{d}$ conformed to the eye-movement instructions, so this group may have been greatly influenced by a small number of atypical responses.

\section{CONCLUDING DISCUSSION}

Discriminability of motion-path extents and the separation between successively presented points was at least as good as the discriminability of extents that did involve memory. In addition, nothing in the data suggests that the type of eye movements, whether pursuit or saccadic, or the absence of eye movements contributed significantly to the perception of extent. Although the experiments did not include continuous test extents, it is a fair inference that the results for such extents would not have differed from the results for the motion-path extents.

The results of our experiments have implications for the analysis of the perception of velocity and extent:

\section{Perception and Velocity}

In the introduction, an account of velocity perception was described which attributed perceived velocity to the operation of an algorithm, $V^{\prime}=$ $S^{\prime} / T^{\prime}$. As we noted, the plausibility of this account depends in part on the premise that motion-path extents are readily discriminable. The present experiments contribute the required demonstration. Of course, this demonstration in no way constitutes proof of the algorithm. It is possible that both $\mathrm{S}^{\prime}$ and $\mathrm{V}^{\prime}$ are determined by parallel processes operating on related input. For example $S^{\prime}$ might be determined by perceived distance $\left(D^{\prime}\right)$ and angular extent of the motion-path $(\theta)$ in a manner analogous to the size-distance algorithm for continuous extents: $S^{\prime}=\theta \times D^{\prime}$. At the same time, $V^{\prime}$ might be determined by the interaction of angular velocity (A) and perceived distance, $V^{\prime}=A \times D^{\prime}$ (Wist, Diener, \& Dichgans, 1976). Although our data cannot substantiate the algorithm, $V^{\prime}=S^{\prime} / T^{\prime}$, the data do satisfy a prerequisite of the algorithm.

The algorithm account of the constancy of perceived velocity is based on the assumption of constancy of perceived extent of the motion path. Our experiments were not designed to provide a systematic assessment of this constancy. Nevertheless, the results of Experiment 1 do suggest the operation of a constancy mechanism. Since the standard displays were half again as far from the subjects as the comparison display, the veridical matches imply the operation of a constancy mechanism which takes distance into account and which could insure constancy.

\section{Perception of Extent}

The data provide clear evidence that discrimination of extent does not depend on the availability of a continuous retinal extent or of an interspace which is demarcated by simultaneously present demarcation points. Since discrimination in our case was so good, we may reasonably doubt that continuous extents would have been discriminated more accurately. If, as implied by this claim, the availability of a continuous retinal subtense contributes little to perception of extent, then what is the information that determines discrimination? One answer is suggested by Rock's (1975) and Rock and Halper's (1969) analysis of the role of the retinal image in determining perceived form. Rock concluded that perceived shape reduces to a set of perceived relative egocentric locations and that the only advantage conferred by a continuous retinal shape is that it represents the set of egocentric locations simultaneously. Rock's experiments showed that a shape will be experienced without a retinal image as long as the points making up the contour of the shape are presented in a way which allows them to be per- 
ceived as a related set of points occupying the correct egocentric locations. Rock's argument may be extended to the present case by proposing that the perception of extent in our experiments fundamentally is the perception of the relative egocentric locations of the endpoints. What our experiments show is that it matters little how the endpoints are demarcated, whether by a single moving point or by a pair of points presented successively or simultaneously. Of course, if the interstimulus interval is too long, or the velocity too slow, effects of memory are likely to appear and continuous extents will show an advantage. However, on the view under consideration, the advantage will be due to the memorial advantage and not to continuity per se. The general implication of these speculations is that analysis of perceived extent should be broadened to include the processing of relative egocentric location as well as the processing of retinal subtenses.

In appraising our experimental procedures with respect to this latter interpretation, Wayne Shebilske (Note 1) expressed an important reservation concerning the condition which required saccadic inspection of two successively exposed points. Shebilske argued that if the subject maintained his direction of gaze after the offset of the previously fixated point and delayed initiation of the saccade until onset of the second point, then the information for extent discrimination could have been provided by the retinal location of the second point, i.e., its spatial separation from the fovea. In this sense, the successive condition might have provided an interspace for judgment, as did the simultaneous condition. On this interpretation, the successive condition would also have been like the simultaneous condition in not imposing a memory load. Given this possibility, the comparison between the successive and simultaneousendpoint conditions cannot yield unambiguous support for the egocentric location interpretation of estimated extent. A supplementary experiment was conducted to assess this argument.

\section{Supplementary Experiment}

Six subjects provided magnitude-estimations of the six pairs of spatially separated, successively presented points under conditions which duplicated Condition c of Experiment 2 in all respects but one. Under the control of a PDP-11/10 computer, the onset of the to-be-saccaded-to point was made contingent on the occurrence of the saccade. The onset of the point was delayed until after the eye had moved to a location midway between the two locations. The sequence on a trial was as follows: The right-hand point was exposed for $1 \mathrm{sec}$ and was fixated by the subject. Following offset of the right-hand point, the subject was required to initiate a saccade promptly, and the computer withheld presentation of the left-

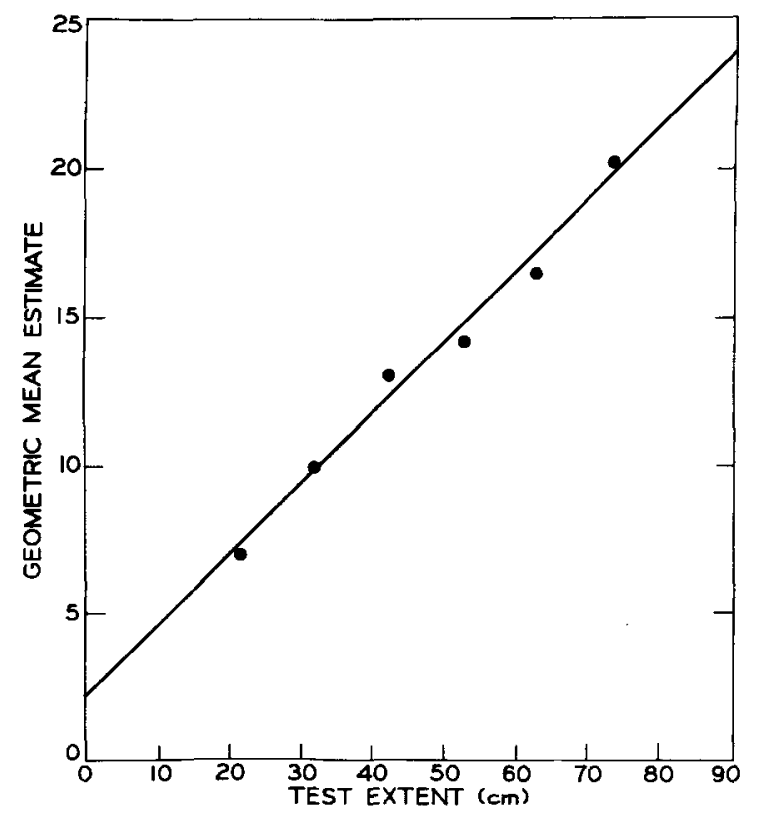

Figure 4. Geometric mean estimates of extent for memory/ saccadic condition where onset of endpoints is contingent on saccadic eye movements.

hand point until a predetermined time had elapsed following the arrival of the eye at a location halfway between the locations of the points. The left-hand point was exposed for $1 \mathrm{sec}$. The trial ended with a 1 -sec reexposure of the right-hand point contingent on occurrence of the prescribed rightward saccade. This procedure made the retinal spatial value an unreliable or misleading cue for the discrimination of extent. Under these conditions, discriminability should deteriorate if it is dependent exclusively on the spatial values of retinal locations. On the other hand; the change of procedure should not affect discrimination of extent if discrimination is based on the difference between egocentric locations.

The mean latency of the leftward saccade was $390 \mathrm{msec}$, and the mean latency of the rightward (return) saccade was $311 \mathrm{msec}$.

The geometric mean estimates for the six test extents are plotted in Figure 4. The function shown in Figure 4 does not differ appreciably from the function obtained under Condition $c$ of Experiment 2. Plainly, the discriminability of extent is not based on the spatial values of retinal locations.

\section{REFERENCE NOTE}

1. Shebilske, W. Personal communication, November 20, 1976.

\section{REFERENCES}

Brown, J. F. The visual perception of velocity. Psychologische Forschung, 1931, 14, 199-232.

EPSTEIN, W. (Ed.). Stability and constancy in visual perception: 
Mechanisms and processes. New York: Wiley-Interscience, 1977. Rock, I. An introduction to perception. New York: Macmillan, 1975.

Rock. I.. \& HALPER. F. Form perception without a retinal image. American Journal of Psychology, 1969. 82, 425-440.

Rock, I.. Hill, A. L., \& Fineman, M. Speed constancy as a function of size constancy. Perception \& Psychophysics. 1968. 4, 37-40.

Stevens, S. S. Psychophysics: Introduction to its perceptual, neural, and social prospects. New York: Wiley-Interscience, 1975.

Wist. E. R.. Diener. H. C., \& Dichgans, J. Motion constancy dependent upon perceived distance and the spatial frequency of the stimulus pattern. Penception \& Psychophysics, 1976. 19. 485-491.

(Received for publication February 4, 1977; revision accepted May 21, 1977.) 